\title{
Generalizations of Melin's inequality to systems
}

\author{
Raymond Brummelhuis
}

\begin{abstract}
We discuss a recent necessary and sufficient condition for Melin's inequality for a class of systems of pseudodifferential operators.
\end{abstract}

\section{Introduction}

Let $V$ be a finite dimensional Hermitian vectorspace, and let $L_{H}(V)$ be the space of Hermitian operators on $V$. We will be interested in lower bounds for pseudodifferential operators with symbols in the space $S_{p h g}^{m}\left(T^{*} \mathbb{R}^{n}, L_{H}(V)\right)$ of polyhomogeneous (or classical) $L_{H}(V)$-valued symbols. Recall that a $C^{\infty}$-function $A: T^{*} \mathbb{R}^{n}$ is in $S_{p h g}^{m}\left(T^{*} \mathbb{R}^{n}, L_{H}(V)\right)$ iff $A$ can be written as an asymptotic sum

$$
A(x, \xi) \simeq A_{m}(x, \xi)+A_{m-1}(x, \xi)+A_{m-2}(x, \xi)+\cdots
$$

with $A_{m-j}(x, \xi)$ homogeneous of degree $m-j$ in $\xi$ for $|\xi| \geq 1 / 2$, say. To such a symbol $A$ we can associate the operator $A^{w}=A^{w}(x, D)$ defined by Weyl quantisation:

$$
A^{w}(x, D) u(x)=(2 \pi)^{-n} \int_{\mathbb{R}^{n}} A\left(\frac{x+y}{2}, \xi\right) u(y) e^{i<x-y, \xi>} d \xi,
$$

acting on, for example, the space $C_{c}^{\infty}\left(\mathbb{R}^{n} ; V\right)$ of $V$-valued $C^{\infty}$ functions on $\mathbb{R}^{n}$. We will denote by $\Psi_{p h g}^{m}\left(\mathbb{R}^{n}, L(V)\right)$ the set of all such Weyl operators with symbols in $S_{p h g}^{m}\left(T^{*} \mathbb{R}^{n}, L_{H}(V)\right)$. One of the advantages of using the Weyl calculus is that all operators in this set are formally self-adjoint.

If $\operatorname{dim}(V)=1$, that is, if we are dealing with scalar symbols and operators, we will systematically denote them by lower case letters $a=a(x, \xi), a^{w}=a^{w}(x, D)$, to distinguish from the systems case.

We will be interested in generalizing Melin's inequality to systems. We begin by recalling Melin's theorem (Melin [12] ; cf. also [8], [10]): 
Theorem 1.1. Let $a \in S_{\text {phg }}^{m}\left(T^{*} \mathbb{R}^{n} ; \mathbb{C}\right)$ be a scalar symbol such that $a_{m}(x, \xi) \geq 0$ on $\mathbb{R}^{n}$. Then

$$
\forall \varepsilon>0 \exists C_{\varepsilon} \forall u \in C_{c}^{\infty}\left(\mathbb{R}^{n}\right):\left(a^{w}(x, D) u, u\right) \geq-\varepsilon\|u\|_{((m-1) / 2)}^{2}+C_{\varepsilon}\|u\|_{((m / 2)-1)}^{2}
$$

if and only iff for all $\left(x_{0}, \xi_{0}\right)$ in $T^{*} \mathbb{R}^{n}$,

$$
a_{m}\left(x_{0}, \xi_{0}\right)=0 \Rightarrow \frac{1}{2} \operatorname{Tr}^{+} Q_{\left(x_{0}, \xi 0\right)}\left(a_{m}\right)+a_{m-1}\left(x_{0}, \xi_{0}\right) \geq 0
$$

Here $Q_{\left(x_{0}, \xi_{0}\right)}\left(a_{m}\right)$ denotes the Hessian of $a_{m}$ in $\left(x_{0}, \xi_{0}\right)$, considered as a quadratic form on the tangent space. The $\mathrm{Tr}^{+}$of such a form $Q=Q_{\left(x_{0}, \xi 0\right)}\left(a_{m}\right)$ on $W=$ $T_{\left(x_{0}, \xi_{0}\right)} T^{*} \mathbb{R}^{n} \simeq T^{*} \mathbb{R}^{n}$ can be defined either purely algebraicly or analytically, which is a point to keep in mind when looking for generalizations. The algebraic definition (which precedes the statement of Melin's theorem in text books, like Hörmander [10], Taylor [14]) is the following: let $F_{Q}: W \rightarrow W$ is the Hamiltonian map associated to $Q: \sigma\left(w, F_{Q}(v)\right)=Q(v, w)$ (where the right hand side is the symmetric bilinear form associated to $Q)$. Then

$$
\operatorname{Tr}^{+} Q:=\sum_{\substack{\mu>0, i \mu \text { eigenvalue of } F_{Q}}} \mu,
$$

For our purposes the analytic definition will turn out to be more usefull : it simply states that

$$
\operatorname{Tr}^{+} Q=\inf \operatorname{Spec} Q^{w}\left(y, D_{y}\right),
$$

where $Q^{w}\left(y, D_{y}\right)$ is the Weyl quantisation of the quadratic form $Q(y, \eta)$ in arbitrary linear symplectic coordinates on $W$. (We recall that operators associated to different symplectic linear coordinates are unitarily equivalent, by the metaplectic invariance of the Weyl-calculus ; cf. for example theorem 18.5.9 in [10]). So an equivalent formulation of Melin's theorem 1.1 is that (1) is equivalent to the non-negativity of the principal symbol $A_{m}$ and of all model operators $Q_{\left(x_{0}, \xi_{0}\right)}\left(a_{m}\right)^{w}+a_{m-1}\left(x_{0}, \xi_{0}\right)$, for characteristic $\left(x_{0}, \xi_{0}\right)$.

A word about terminology: we will call inequality (1), by itself, Melin's inequality, although in the litterature the term "Melin's inequality" is often used for the whole of theorem 1.1, that is, for the equivalence of (1) with (2).

Remark 1.2. Melin's inequality can be looked upon as being, in some sens, the weakest possible improvement of the sharp Gårding inequality (Hörmander [5], Lax and Nirenberg [11]), which is basically (1) for a fixed, $a^{w}$-dependend $\varepsilon$, under the sole hypothesis of non-negativity of $a_{m}$. For scalar operators other, stronger generalizations are known, for example Hörmanders 6/5-th inequality (Hörmander [9]), the Fefferman-Phong inequality and the SAK-principle (cf. Fefferman [4] and it's references, and, more recently, Heraut [5]). These will not be considered here and we only note that their correct generalization to systems is a difficult problem.

It is easy to show that the necessary condition for Melin's inequality immediately generalizes to systems. If $A^{w} \in \Psi_{p h g}^{m}\left(\mathbb{R}^{n}, L_{H}(V)\right)$, let

$$
\Sigma\left(A_{m}\right):=\left\{(x, \xi) \in T^{*} \mathbb{R}^{n}: \operatorname{Ker} A_{m}(x, \xi) \neq 0\right\}
$$


be the characteristic set of the system $A^{w}$ and, for an $\left(x_{0}, \xi_{0}\right) \in \Sigma\left(A_{m}\right)$, let $\Pi_{\left(x_{0}, \xi_{0}\right)}$ be the orthogonal projection of $V$ onto $\operatorname{Ker} A_{m}\left(x_{0}, \xi_{0}\right)$. If $A_{m}(x, \xi) \geq 0$ pointwise (as an Hermitian operator on $V$ ), then

$$
Q_{\left(x_{0}, \xi_{0}\right)}\left(A_{m}\right)=\text { Hessian in }\left(x_{0}, \xi_{0}\right) \text { of } \Pi_{\left(x_{0}, \xi_{0}\right)} A_{m}(x, \xi) \Pi_{\left(x_{0}, \xi_{0}\right)},
$$

will be an invariantly defined $L_{H}(V)$-valued quadratic form on $T_{\left(x_{0}, \xi_{0}\right)}\left(T^{*} \mathbb{R}^{n}\right)$, with values in the non-negative operators in $L_{H}(V)$. We then have that

$$
\begin{gathered}
A^{w} \text { satisfies Melin's inequality }(1) \Rightarrow A_{m} \geq 0 \text { and } \\
\forall\left(x_{0}, \xi_{0}\right) \in \Sigma\left(A_{m}\right): \frac{1}{2} Q_{\left(x_{0}, \xi_{0}\right)}\left(A_{m}\right)^{w}\left(y, D_{y}\right)+\Pi_{0} A_{m-1}\left(x_{0}, \xi_{0}\right) \Pi_{0} \geq 0,
\end{gathered}
$$

as a densely defined operator on $L^{2}\left(\mathbb{R}^{n} ; V\right)$.

We note in passing that there exist simple $2 \times 2$ systems $A^{w}=A_{2}^{w}$, homogeneous of order 2 and with $A_{2} \geq 0$, such that at some points of the characteristic set, $Q_{\left(x_{0}, \xi_{0}\right)}\left(A_{m}\right)^{w}$ is not non-negative (cf. [5], or [1], [2]), in marked difference to the scalar case.

The necessary condition (4) can be considerably strengthened at points $\left(x_{0}, \xi_{0}\right)$ of the characteristic set for which $A_{m}\left(x_{0}, \xi_{0}\right)$ is not the 0-operator (Hörmander [8] if the kernel has dimension at most 1, Brummelhuis [1], [2] in general). To state this new condition, we will write $\Pi_{0}=\Pi_{\left(x_{0}, \xi_{0}\right)}$, to simplify notations. We first note that

$$
A_{m}^{\#}\left(x_{0}, \xi_{0}\right): \operatorname{Im} A_{m}\left(x_{0}, \xi_{0}\right)=\left(\operatorname{Ker} A_{m}\left(x_{0}, \xi_{0}\right)\right)^{\perp} \rightarrow \operatorname{Im} A_{m}\left(x_{0}, \xi_{0}\right)
$$

is a well-defined isomorphism of vectorspaces. Next, by non-negativity of $A_{m}$, $\Pi_{0} d A_{m} \Pi_{0}=0$ in $\left(x_{0}, \xi_{0}\right)$, which easily implies that

$$
L_{\left(x_{0}, \xi_{0}\right)}:(y, \eta) \rightarrow\left(A_{m}^{\#}\left(x_{0}, \xi_{0}\right)\right)^{-1 / 2}\left\langle d A_{m}\left(x_{0}, \xi_{0}\right),(y, \eta)\right\rangle \Pi_{0}
$$

is a well-defined operator-valued linear form. The strengthened necessary condition for Melin's inequality then is:

Theorem 1.3. Suppose that $A^{w}$ satisfies Melin's inequality (1). Then $A_{m} \geq 0$ and for all $\left(x_{0}, \xi_{0}\right) \in \Sigma\left(A_{m}\right)$,

$$
\frac{1}{2} Q_{\left(x_{0}, \xi_{0}\right)}\left(A_{m}\right)^{w}\left(y, D_{y}\right)+\Pi_{0} A_{m-1} \Pi_{0}-L_{\left(x_{0}, \xi_{0}\right)}^{w}\left(y, D_{y}\right)^{*} L_{\left(x_{0}, \xi_{0}\right)}^{w}\left(y, D_{y}\right) \geq 0 .
$$

The proof of this theorem is based on a micro-local decoupling argument near a given point $\left(x_{0}, \xi_{0}\right)$ of the characteristic set: one shows that one can find, microlocally, an orthogonal projection-valued symbol in $S_{p h g}^{0}\left(T^{*} \mathbb{R}^{n}, L_{H}(V)\right)$, commuting with the principal symbol $A_{m}$, such that $\Pi\left(x_{0}, \xi_{0}\right)$ is the orthogonal projection onto the kernel of $A_{m}\left(x_{0}, \xi_{0}\right)$ and such that $(I-\Pi) A_{m}(I-\Pi)$ is elliptic in $\left(x_{0}, \xi_{0}\right)$. One then easily proves that, again micro-locally, $A^{w}$ satisfies Melin's inequality iff $\Pi^{w} A^{w} \Pi^{w}$ does, and the theorem follows by applying (4) to the latter. Hörmander [8] carried out this argument under the additional hypotheses that $\Sigma\left(A_{m}\right)$ is smooth and that $A_{m}$ is of constant $\operatorname{rank} N-1$ on the characteristic set, where $N=\operatorname{dim}(V)$, and stated (5) in a form which does not easily generalize to more singular systems.

To go now in the other direction, and find sufficient conditions for (1), one can proceed in several ways: 
- Faithfully modelling one's approach on the one used in the scalar case, one can start by trying to compute the spectrum, or at least the infimum of the spectrum, of the model operators (4), (5).

In fact, it suffices, by the proof of theorem 1.3, to understand the spectrum of the model operators in (4), which are of the form

$$
\sum_{j, k=1}^{n} A_{j k} D_{j} D_{k}+B_{j k}\left(x_{j} D_{k}+D_{k} x_{j}\right)+C_{j k} x_{j} x_{k}+E,
$$

where $D_{j}=i^{-1} \partial / \partial x_{j}$, with $A_{j k}=A_{k j}^{*}, B_{j k}, C_{j k}=C_{k j}^{*}$ and $E$ all in $L_{H}(V)$, and with principal symbol (in $S\left(\langle(x, \xi),\rangle^{2},\left(\langle(x, \xi)\rangle^{-2}\left(|d x|^{2}+|d \xi|^{2}\right) ; L_{H}(V)\right)\right.$ ) non-negative, in the sense of Hermitian operators:

$$
\left(\left(\sum_{j k} A_{j k} \xi_{j} \xi_{k}+2 B_{j k} x_{j} \xi_{k}+C_{j k} x_{j} x_{k}\right) v, v\right) \geq 0, \quad \forall v \in V .
$$

By analogy with the scalar case, such operators were called harmonic oscillator systems or, more briefly, matrix oscillators, in [1], [2].

However, diagonalizing (6) is a very hard problem, even in the simplest non-scalar case of $n=1$ and $V=\mathbb{C}^{2}$ : for example, as mentioned above, the non-negativity condition (7) is not sufficient for (6) to be non-negative, even if $E \geq 0$ (cf. [9]). There are some examples for which the spectrum can be computed explicitly (cf. the references of [2]), but nothing conclusive seems to be known.

In [1], [2] we gave a general lower bound for certain subclasses of matrix oscillators, which included all matrix oscillators in dimension 1 , that is, with $n=1$. This lower bound, which is computed as the solution of a certain auxilliary max-min problem in a space of $n N \times n N$-matrices, is sharp in the trivial case of simultaneously diagonalizable coefficients, but unfortunately almost never in general. However, being a lower bound, one might hope to use it for a sufficient (though not necessarily necessary) condition for Melin's inequality for systems. This was the approach of [1], [2]. We only wish to note here that to succeed it was necessary to impose certain structural conditions on the principal symbol, regarded purely as a matrix valued $C^{\infty}$ function, without it being clear if such conditions were really necessary, or just an artifact of the proof. A similar phenomenon will be encountered below. The conditions of [1], [2] are in general difficult to verify, but examples are known. For example, the "weak Morse" condition from [2] is automatically satisfied by transversally elliptic systems.

- One can try to circumvent the problem of analyzing the spectrum of these matrix oscillators, by proving directly that non-negativity of the model operators (5) is also sufficient for Melin's inequality.

A first result along these lines can be found, among other Gårding inequalities for systems, in Parenti and Parmeggiani [13], who proved the sufficiency of (5) under the hypotheses that $\Sigma\left(A_{m}\right)$ is a symplectic submanifold, $A_{m}$ is of constant rank $r$ along $\Sigma\left(A_{m}\right)$, and that $\operatorname{det} A_{m}$ vanishes of order exactly $2(N-r)$ on $\Sigma\left(A_{m}\right), N$ 
being the dimension of $V$. We will encounter a significant generalisation of this result below, where it will turn up as a corollary of yet another approach to Melin's inequality, which is the next one:

- Thirdly, acting on a suspicion that non-negativity of the model operators (5) might not always suffice, one might seek to enlarge the class of the latter.

This is the approach we will explain here. Adapting an idea from Hörmander [7] (cf. also [10], section 22.4) we will replace the single operator in (5) by a family of second order operators.

\section{Main results}

The precise definition of the family of model operators by which we will replace the single operator in (5) is the following:

Definition 2.1. For $\left(x_{0}, \xi_{0}\right) \in \Sigma\left(A_{m}\right)$ we define $\mathfrak{Q}_{\left(x_{0}, \xi_{0}\right)}(A)$ to be the set of all quadratic polynomials $Q=Q(y, \eta)$ with coefficients in $L_{H}(V)$ for which there exist sequences $\left(x_{\nu}, \xi_{\nu}\right) \rightarrow\left(x_{0}, \xi_{0}\right), \lambda_{\nu} \rightarrow \infty, \lambda_{\nu} \geq 0$ and orthogonal projections $\Pi_{\nu} \in$ $L_{H}(V)$, commuting with $A_{m}\left(x_{\nu}, \xi_{\nu}\right)$, such that

$$
Q(y, \eta)=\lim _{\nu \rightarrow \infty} \lambda_{\nu}^{1-m} \Pi_{\nu} A\left(x_{\nu}+\lambda_{\nu}^{-1 / 2} y, \lambda_{\nu} \xi_{\nu}+\lambda_{\nu}^{1 / 2} \eta\right) \Pi_{\nu},
$$

pointwise for $(y, \eta) \in T^{*} \mathbb{R}^{n}$.

The only difference with the analogous definition in [7] is the inclusion of the projections $\Pi_{\nu}$; as we will try to explain below, this imposes itself by the proof.

A moment's thought shows that, assuming that $\Pi_{\nu}$ to converge to some orthogonal projection $\Pi_{\infty}$ (as we may do wlog), then

$$
\begin{aligned}
Q= & \Pi_{\infty} Q_{\left(x_{0}, \xi_{0}\right)}\left(A_{m}\right)(y, \eta) \Pi_{\infty}+\lim _{\nu \rightarrow \infty} \lambda_{\nu}^{1 / 2} \Pi_{\nu}\left\langle d_{x, \xi} A_{m},\left(y_{\nu}, \eta_{\nu}\right)\right\rangle+ \\
& \lim _{\nu \rightarrow \infty} \lambda_{\nu} \Pi_{\nu} A_{m}\left(x_{\nu}, \xi_{\nu}\right) \Pi_{\nu}+\Pi_{\infty} A_{m-1}\left(x_{0}, \xi_{0}\right) \Pi_{\infty},
\end{aligned}
$$

where the Hessian $Q_{\left(x_{0}, \xi_{0}\right)}\left(A_{m}\right)$ was defined in (3) above.

Our main result now is that, together with non-negativity of the principal symbol, non-negativity of all $Q^{w}$ in $\mathfrak{Q}_{\left(x_{0}, \xi_{0}\right)}(A)$ is necessary and sufficient for Melin's inequality:

Theorem 2.2. (Brummelhuis and Nourrigat [3], theorem 1.3 and corollary 2.3) Let $A^{w} \in \Psi_{p h g}^{m}\left(\mathbb{R}^{n}, L_{H}(V)\right)$. Then

(i) If $A^{w}$ satisfies Melin's inequality (1), then $A_{m} \geq 0$ and $Q^{w} \geq 0$ for all $Q \in$ $\mathfrak{Q}_{\left(x_{0}, \xi_{0}\right)}(A),\left(x_{0}, \xi_{0}\right) \in \Sigma\left(A_{m}\right)$.

(ii) Conversely, suppose that the principal symbol is non-negative and satisfies the following condition: given $\left(x_{0}, \xi_{0}\right) \in \Sigma\left(A_{m}\right)$ there exists a constant $C>0$ and a neighborhood of $\left(x_{0}, \xi_{0}\right)$ such that, for $(x, \xi)$ in this neighborhood and for $v$ any eigenvector of $A_{m}(x, \xi)$,

$$
\left\|d_{x, \xi} A_{m}(x, \xi) v\right\|^{2} \leq C\left(A_{m}(x, \xi) v, v\right) .
$$

Then the necessary condition in (i) is also sufficient. 
A condition which implies (9), and which is somewhat easier to state, is that there exists a constant $C>0$ and a neighborhood such that, pointwise in this neighborhood (and with a slightly imprecise notation),

$$
d_{x, \xi} A_{m}(x, \xi)^{2} \leq C A_{m}(x, \xi),
$$

in the sense of Hermitian operators on $V$. Note that this is always satisfied by non-negative scalar functions (as is the condition of being "weak Morse" from [2]), and therefore also by diagonal non-negative matrix functions (contrary being "weak Morse").

We do not know whether the condition (9) on the principal symbol is really necessary or not. What is easy to see is that it is not satisfied by all matrix-valued functions, not even of a single variable: a counter example is given by the function

$$
z \rightarrow\left(\begin{array}{cc}
z^{2} & z \\
z & 1
\end{array}\right), \quad z \in \mathbb{R}
$$

Since this example is $C^{\infty}$ diagonalizable, it also shows that (9) is not invariant with respect to conjugation by a unitary matrix function.

Apart from having to impose this condition (9), another inconvenient of theorem 2.2 is that it might be difficult, for a given operator $A^{w}$, to determine the set $\mathfrak{Q}_{\left(x_{0}, \xi_{0}\right)}(A)$. Happily, there exists a situation where we can both replace this set by a single second order operator, and where we do not need to check (9):

Theorem 2.3. ([3], theorem 3.1) Let $N=\operatorname{dim} V$ and let $A^{w} \in \Psi_{p h g}^{m}\left(\mathbb{R}^{n}, L(V)\right)$ be such that:

(i) The rank of $A_{m}$ is constant on the characteristic set: $\operatorname{rank}\left(A_{m}\right) \equiv r$ on $\Sigma\left(A_{m}\right)$.

(ii) The determinant of $A_{m}$ vanishes to order exactly $2(N-r)$ on $\Sigma\left(A_{m}\right)$, in the sense that for each compact $K$ there exists constants $C_{1}, C_{2}>0$ such that if $d(x, \xi)$ is the distance of $(x, \xi)$ to $\Sigma\left(A_{m}\right)$, then

$$
C_{1} d(x, \xi)^{2(N-r)} \leq \operatorname{det}\left(A_{m}(x, \xi)\right) \leq C_{2} d(x, \xi)^{2(N-r)}, \quad(x, \xi) \in K .
$$

Then $A^{w}$ satisfies Melin's inequality iff (5) holds, for all $\left(x_{0}, \xi_{0}\right) \in \Sigma\left(A_{m}\right)$.

This theorem generalizes the result of Parenti and Parmeggiani [13] quoted above. Note that we do not make any assumption on the structure of $\Sigma\left(A_{m}\right)$ anymore.

\section{Proofs}

We will limit ourselves to some remarks on the proofs of theorems 2.2 and 2.3, which will hopefully help to clarify the origen of the projections $\Pi_{\nu}$ in definition 2.1 , and of the condition (9).

The proof of theorem 2.2 is in fact faithfully modelled on Melin's original proof in [12]: one can use his $S_{1 / 2,1 / 2}^{0}$-partition of unity arguments to reduce the sufficiency of 2.2(i) to the following statement, where we will suppose, for simplicity (and without essential loss of generality), that $A_{m-1}=0$, and where we write $A_{m(\beta)}^{(\alpha)}$ for $\partial_{\xi}^{\alpha} \partial_{x}^{\beta}$ : 
Claim 3.1. Suppose that there exist $\rho_{0}>0$, sequences $\left(x_{\nu}, \xi_{\nu}\right)$ in a compact of $T^{*} \mathbb{R}^{n} \backslash 0, \lambda_{\nu} \rightarrow \infty$ and $v_{\nu} \in C_{c}^{\infty}(B(0,1))$ such that

$$
\left(\left(\sum_{|\alpha|+|\beta| \leq 2} \lambda^{1-(|\alpha|+|\beta|) / 2} A_{m(\beta)}^{(\alpha)}(x, \xi) \frac{y^{\beta} \eta^{\alpha}}{\alpha ! \beta !}\right)^{w}\left(y, D_{y}\right) v, v\right) \leq-\rho_{0}\left\|v_{\nu}\right\|_{(4)}^{2}
$$

(the norm on the right being a Sobolev norm). Then there exists a $Q \in \mathfrak{Q}_{\left(x_{0}, \xi_{0}\right)}(A)$ such that $Q^{w}$ is not non-negative.

To prove the claim, one first shows that $\lambda_{\nu} A_{m}\left(x_{\nu}, \xi \nu\right)$ and $\left\|v_{\nu}\right\|_{(4)}$ stay bounded. By compactness, we can then assume that $v_{\nu} \rightarrow v_{0}$ in the Sobolev space of order 3 , with $v_{0} \neq 0$ (e.g. since we can assume all $v_{\nu}$ to have $L^{2}$-norm equal to 1 ). We can also assume that $\left(x_{\nu}, \xi_{\nu}\right) \rightarrow\left(x_{0}, \xi_{0}\right)$. By the above,

$$
\left(A_{m}\left(x_{\nu}, \xi_{\nu}\right) v_{0}, v_{0}\right) \rightarrow 0
$$

Now in the systems case this does not necessarily imply that $A_{m}\left(x_{0}, \xi_{0}\right)=0$. However, using the fact that $A_{m}(x, \xi)$ can be continuously diagonalized in a neighborhood of $\left(x_{0}, \xi_{0}\right)$, one can construct a sequence of orthogonal projections $\Pi_{\nu} \rightarrow \Pi_{\infty}$ such that

$$
\lambda_{\nu} \Pi_{\nu} A_{m}\left(x_{\nu}, \xi_{\nu}\right) \Pi_{\nu}
$$

stays bounded. In fact, $\Pi_{\nu}$ will project exactly onto the span of those eigenvectors of $A_{m}\left(x_{\nu}, \xi \nu\right)$ for which the component of $v_{0}$ along that eigenvector does not tend to 0 as $\nu \rightarrow \infty$; in particular, $\Pi_{\infty} v_{0}=v_{0}$. One next shows that this sequence of $\Pi_{\nu}$ 's can be used to construct an element $Q$ of $\mathfrak{Q}_{\left(x_{0}, \xi_{0}\right)}\left(A_{m}\right)$. The final step of the proof then is to show that

$$
\left(Q^{w} v_{0}, v_{0}\right)<0
$$

This is not a trivial consequence of the hypothesis of the claim, since we need to show that we can replace $v_{\nu}$ by $\Pi_{\nu} v_{\nu}$ in (10), and it is in the verification of this point (and only there) that we need condition (9). We refer to [3] for details.

Turning next to theorem 2.3, it's proof uses theorem 2.2, but instead of applying the latter directly, we apply it to the operators $\Pi^{w} A^{w} \Pi^{w}$ from the proof of theorem 1.3. Using the constancy of rank assumption (i), the principal symbol of the latter vanishes on $\Sigma\left(A_{m}\right)$. We can then reduce theorem 2.3 to the special case where $r=0$, basically since we can reduce Melin's inequality for $A^{w}$ to Melin's inequality for $\Pi^{w} A^{w} \Pi^{w}$. Condition 2.3(ii) then becomes a transversal ellipticity condition, which can be used to show that $A_{m}$ satisfies (9) and that (5) (which, in this case, is just (4)) implies positivity of all $Q^{w}, Q \in \mathfrak{Q}_{\left(x_{0}, \xi_{0}\right)}(A)$. An appeal to theorem 2.2 then finishes the proof.

\section{References}

[1] R. G. M. Brummelhuis, Sur les inégalités de Gårding pour les systèmes d'opérateurs pseudo-différentiels, C. R. Acad. Sci. Paris Série I, 315, 149 152 (1992). 
[2] R. G. M. Brummelhuis, On Melin's inequality for systems, to appear in Comm. PDE.

(Earlier versions were available as CWI Report AM-R9111 (1991) and Univ. de Reims prépublication 97.07 (1997). )

[3] R. G. M. Brummelhuis and J. Nourrigat, A necessary and sufficient condition for Melin's inequality for a class of systems, Univ. De Reims prépublication 00.09 (2000), to appear in J. Analyse Math.

[4] C. Fefferman, The uncertainty principle, Bull. Amer. Math. Soc. 9, 129 - 206 (1983).

[5] F. Hérau, Opérateurs pseudo-différentiels semi-bornés, Thèse, Université de Rennes I (1999).

[6] L. Hörmander, Pseudo-differential operators and non-elliptic boundary problems, Ann. Math. 83, 129 - 209 (1966).

[7] L. Hörmander, A class of hypoelliptic pseudo-differential operators with double characteristics, Math. Ann. 217, 165 - 188 (1975).

[8] L. Hörmander, The Cauchy problem for differential operators with double characteristics, J. Analyse Math. 32, 118 - 196 (1977).

[9] L. Hörmander, The Weyl calculus of pseudo-differential operators, Comm. Pure Appl. Math. 32, 359 - 443 (1979).

[10] L. Hörmander, The analysis of linear partial differential operators III, Grundlehren der mathematischen Wissenschaften 274, Springer-Verlag, Berlin - Heidelberg, 1985.

[11] P. D. Lax and L. Nirenberg, On stability for difference schemes: a sharp form of Gårdings inequality, Comm. Pure Appl. Math. 19, 473 - 492 (1966).

[12] A. Melin, Lower bounds for pseudo-differential operators, Ark. Mat. 9, 117-140 (1971).

[13] C. Parenti and A. Parmeggiani, Lower bounds for systems with double characteristics, preprint, 2000, to appear in J. Analyse Math.

[14] M. Taylor, Pseudodifferential operators, Princeton Mathematical Series 34, Princeton University Press, Princeton, New Jersey, 1981.

Université De Reims, Département de mathématiques, BP 1039, 51687 Reims CÉdex, France.

raymond. brummelhuis@univ-reims.fr 